\title{
Smiling makes you look older
}

\author{
Tzvi Ganel ${ }^{1}$
}

Published online: 9 April 2015

(C) Psychonomic Society, Inc. 2015

\begin{abstract}
People smile in social interactions to convey different types of nonverbal communication. However, smiling can potentially change the way a person is perceived along different facial dimensions, including perceived age. It is commonly assumed that smiling faces are perceived as younger than faces carrying a neutral expression. In the series of experiments reported here, I describe an unintuitive and robust effect in the opposite direction. Across different experimental conditions and stimulus sets, smiling faces were consistently perceived as older compared to neutral face photos of the same persons. I suggest that this effect is due to observer failure to ignore smile-associated wrinkles, mainly along the region of the eyes. These findings point to a misconception regarding the relationship between facial smile and perceived age and shed new light on the processes underlying human age perception.
\end{abstract}

Keywords Face perception $\cdot$ Age evaluations $\cdot$ Facial expression

\section{Introduction}

People smile in social interactions to convey a wide range of nonverbal communications. Indeed, smiling has been shown to result in positive evaluation of the smiling person regarding different social aspects. For example, people who smile are evaluated as friendlier, more attractive (Jones, Debruine,

Tzvi Ganel

tganel@bgu.ac.il

1 Department of Psychology, Ben-Gurion University of the Negev, Beer-Sheva 84105, Israel
Little, Conway, \& Feinberg, 2006; Otta, Lira, Delevati, Cesar, \& Pires, 1994) and are remembered more easily compared with people displaying a neutral expression (Tsukiura \& Cabeza, 2008). In a similar vein, information on peoples' age plays a major role in how they are evaluated in social contexts; younger people are perceived as more attractive, more likable, and more energetic than older people (Ebner, 2008). The purpose of this study was to test whether the positive effects of smiling would transfer to perception of facial age.

Age is rated by observers as one of the primary facial features that they readily extract when viewing unfamiliar faces (George \& Hole, 1998). Yet, the cognitive processes that underlie the perception of age and its relationship with other aspects of the face are poorly understood (Dagovitch \& Ganel, 2010). A number of studies that looked at the processing of various facial dimensions such as expression, identity, gender, and the direction of gaze (Ganel \& Goshen-Gottstein, 2002, 2004; Ganel, Goshen-Gottstein, \& Goodale, 2005; Schweinberger \& Soukup, 1998) suggested that people are unable to process a single aspect of a face without being affected by task-irrelevant information belonging to the same face. It is therefore assumed here that the perception of age would also be affected by task-irrelevant information related to smiles.

One possible consequence of such an effect is that smiling faces would be perceived as younger compared with neutral faces. Such a result would be in line with the commonly held conviction that smiling faces are perceived as younger (Voelkle, Ebner, Lindenberger, \& Riediger, 2012). Conversely, it is also possible that smiling would have the opposite effect on perceived age. In particular, the skin in the eye region is thinner than in the other parts of the face and more likely to be deformed by big face movements such as the contraction of the zygomaticus major during smiling (Root \& Stephens, 2003). Given that the presence of wrinkles leads to evaluations of older age (Mark et al. 1980), and that perceivers are 
unable to ignore task-irrelevant information, it is possible that smiling faces would be perceived to be older rather than younger compared with faces in neutral expression.

Remarkably, only one study has looked at the effects of smiling on perceived age (Voelkle et al. 2012). In this latter study, participants were asked to evaluate age as well as other facial attributes of a series of facial photos of different individuals. Six different photos of each person expressing different emotions were presented throughout the experimental session and subjects were repeatedly asked to evaluate the person's age. The results were inconsistent. Both neutral and smiling faces were perceived as younger compared to faces expressing other emotions, but no significant differences were found between neutral and smiling faces. However, it is difficult to interpret these findings due to the fact that the experimental design included repeated presentations of photos of belonging to the same persons bearing different expressions. This may have biased overt age evaluations. It is possible, for example, that following multiple repetitions of photos of different expressions belonging to the same person (note that all photos of each person were taken during a single session and were identical in actual age), participants could have gained explicit awareness that expression should be taken into account in their age evaluations. This could have biased participants' age evaluations to conform with the commonly held conviction that smiling faces should be perceived as younger, and therefore could mask potential effects of smile on perceived age. To avoid this potential pitfall, the present design did not use multiple presentations of photos belonging to the same person during the same experimental session. Instead, in all experiments, identity was counterbalanced across participants so that each participant was presented with a series of faces that included only a single exemplar photo of each individual.

\section{Experiments 1a-1c}

In Experiment 1, participants evaluated the age of a series of smiling and neutral face photos. To increase external validity, three completely different facial sets were used in Experiments 1a-1c. To avoid irrelevant effects of potential differences between smiling and neutral faces, photos of the same people were presented to all participants (each participant was presented with one instance of each person, carrying either a smiling or a neutral expression) in a counterbalanced design. Would perceived age differ between smiling and neutral faces?

\section{Method}

\section{Participants}

Twenty students from Ben Gurion University of the Negev (five males, mean age $=22.95$ years, $\mathrm{SD}=1.1$ years) participated in Experiment 1a, 20 different participants (five males, mean age $=22.35$ years, $\mathrm{SD}=1.8$ years) participated in Experiment $1 \mathrm{~b}$, and 20 different participants (three males, mean age $=22.85$ years, $\mathrm{SD}=0.9$ years) participated in Experiment $1 \mathrm{c}$. All participants had normal or corrected-tonormal vision and received course credit for their participation. All ethics were approved by the local ethics committee.

\section{Design and materials}

Experiment 1a Photos of 30 women and 30 men, all with neutral and smiling expressions, were taken from the Karolinska Directed Emotional Faces (KDEF) database of faces (Lundqvist, Flykt, \& Öhman, 1998). The average age of the people photographed in the KDEF set was 25 years old. Stimuli were cropped to $18 \times 13 \mathrm{~cm}$ in size and presented on a $17^{\prime \prime}$ screen using E-Prime software.

Experiment 1b Photos of 40 women and 40 men, all with neutral and smiling expressions, were taken from the FACES database (Ebner, Riediger, \& Lindenberger, 2010) and from the PAL face database (Minear \& Park, 2004). These sets included information on the real age of each of the faces. The average age was 24.86 years old and the age range was 20-39 years. Stimuli were cropped to $18 \times 13 \mathrm{~cm}$ in size and presented on a 17" screen using E-Prime software.

Experiment 1c Photos of 37 women and 43 men, all with neutral and smiling expressions, were taken from several internet databases and from a set of Israeli faces collected by the author (Fig. 1a). The face photos did not include information about the real age of the photographed face, but the average perceived age in this set was older compared with the age of the face photos presented in Experiment 1a. In all other aspects, the design was similar to the one used in Experiment 1a.

\section{Experimental procedure}

The procedures used in Experiment 1a-1c were similar, with the exception that a different face database was used in each experiment. The stimulus set was divided into two equal sets (A and B, 30 individuals in each set in Experiment 1a, 40 individuals in each set in Experiments $1 \mathrm{~b}$ and 1c). For onehalf of the participants, the photographs of the people in set A were presented in a smiling expression and those in set $\mathrm{B}$ were presented in a neutral expression. The remaining participants saw the faces from set $\mathrm{A}$ in a neutral expression and the faces from set B in a smiling expression. Participants were told that they would be presented with a series of face photos and were asked to evaluate the age of each face as accurately as possible. The faces from sets A and B were then presented in a random intermixed fashion. Each face was presented on the screen until a response was made. Participants typed their 
a

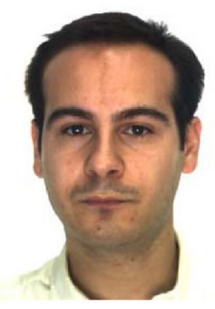

b

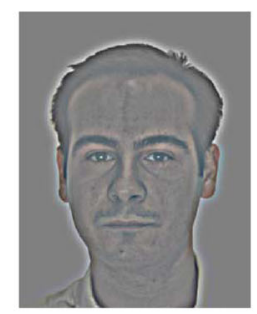

c

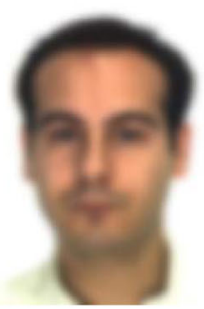

d

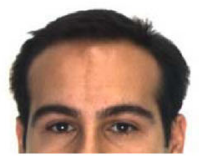

e

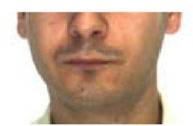

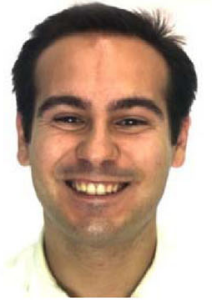
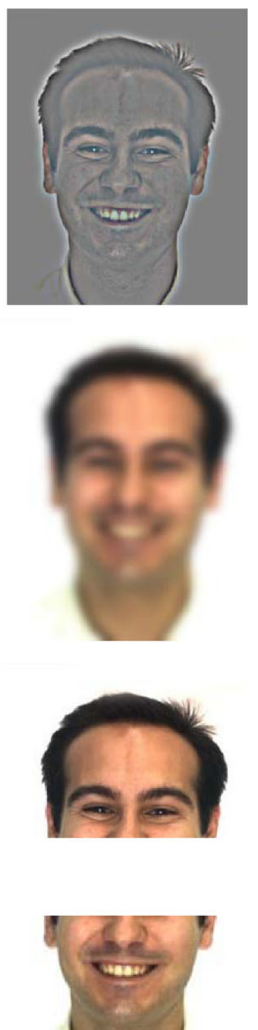

Fig. 1 a-e Sample stimuli used in Experiments 1-3. a Full neutral and smiling faces presented in Experiment 1c. High spatial frequency (b) and low spatial frequency (c) faces presented in Experiments $2 a$ and $2 b$. Cropped photos of the upper (d) and lower (e) parts of the faces used in Experiments $3 \mathrm{a}$ and $3 \mathrm{~b}$. In all experiments, participants were presented with only one exemplar of each person. Expression (neutral vs smiling) was counterbalanced between subjects

evaluated age response, which appeared below the photo of each face until a "Return" key was pressed, which was followed by the presentation of the next stimulus.

\section{Results and discussion}

The effect of smiling on perceived age was calculated for each participant by comparing the average of neutral faces and the average of smiling faces. Due to the fact that the faces of the same people expressing different emotions were presented to all participants in a counterbalanced manner (see Methods), the real age of the faces was not required to calculate the effect of smiling. One- or three-digit age responses were classified as

errors and were removed from the analysis (less than $1 \%$ of the total responses).

As can be seen in Fig. 2, smiling faces were evaluated as older compared with neutral faces in all three experiments. When people smiled, they were perceived as between 1 year to almost 2 years older than when they were presented bearing a neutral expression, depending on the specific facial database. The differences were significant in Experiment 1a $[\mathrm{t}(19)=3.95, P<.001$, partial eta square $=.45]$, in Experiment $1 \mathrm{~b}[\mathrm{t}(19)=3.37, P<.01$, partial eta square $=.37]$, and in Experiment $1 \mathrm{c}[\mathrm{t}(19)=4.43, \mathrm{p}<.001$, partial eta square $=$ $.51]$. These results show, for the first time, that smiling has an aging effect on faces. Unlike previously assumed, smiling faces are perceived as older, not as younger, compared with neutral faces.

Note that information about average age and information about the specific age of each face were available for the sets used in Experiments 1a and 1b, respectively. This allowed calculation of whether the age of the faces within each experiment was perceived as older than the real age, as shown in previous studies for faces of young adults (Voelkle et al. 2012). The average age of the faces presented in Experiment 1a was 25 years old. As can be seen in Fig. 2, both neutral faces and smiling faces were perceived as older than their actual age $[\mathrm{t}(19)=2.79, P<0.05$, and $\mathrm{t}(19)=5.87, P<$ .001 , respectively]. A similar effect was found in Experiment

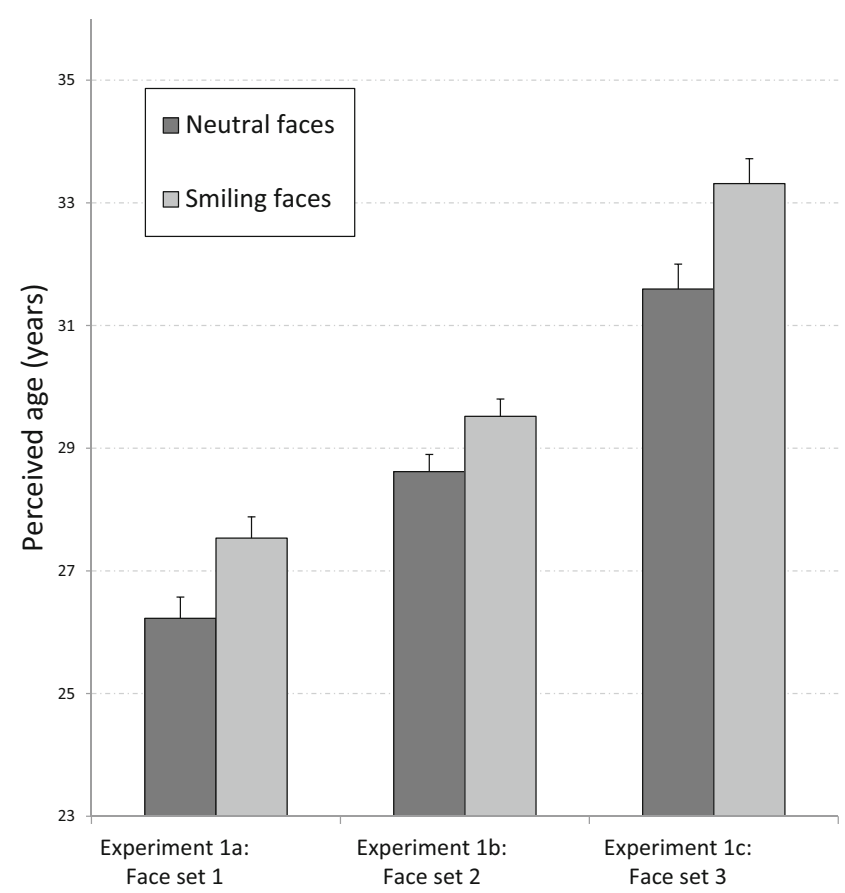

Fig. 2 Average perceived age for smiling and neutral faces in Experiments 1a, 1b, and 1c. Different facial sets were used in each of the three experiments. In all experiments, smiling faces were perceived as significantly older than faces of the same people with a neutral expression. Error bars represent $95 \%$ confidence intervals for withinsubject designs (Jarmasz \& Hollands, 2009) 
2b. Again, both neutral faces and smiling faces were perceived as older than their actual age $[\mathrm{t}(19)=8.39, P<0.01$, and $\mathrm{t}(19)$ $=9.48, P<.001$, respectively].

\section{Experiments 2a-2b}

The results of Experiment 1 show a robust effect of smile on the perceived age. Contrary to accepted wisdom, smiling faces were perceived as older compared with neutral faces. Experiments $2 \mathrm{a}-2 \mathrm{~b}$ were designed to test whether this effect derived from the wrinkles created around the region of the eyes of smiling faces. As discussed, smiling leads to the formation of wrinkles around the eyes, which could account for the extra years of age attributed to smiling faces. To test this idea, I applied a graphic manipulation (spatial frequency filtering) to control for the availability of fine-grained visual information (that includes wrinkles) in Experiments $2 \mathrm{a}$ and $2 \mathrm{~b}$.

\section{Method}

\section{Participants}

Twenty students from Ben Gurion University of the Negev (five male, mean age $=24$ years, $\mathrm{SD}=1.1$ years) participated in Experiment 2a. Twenty different participants (1 male, mean age $=23$ years, $\mathrm{SD}=1.1$ ) participated in Experiment $2 \mathrm{~b}$. One participant from Experiment $2 b$ was removed from the analysis due to her failure to complete the experimental session within a reasonable time frame. Her average reaction times during face judgments were above $21 \mathrm{~s}$-more than three times slower than the average reaction times of the other participants in this experiment. All participants had normal or corrected-to-normal vision and received course credit for their participation.

\section{Design and procedure}

The design and procedure were similar to those used in Experiment 1c. The same stimulus set was used in Experiments $2 \mathrm{a}-2 \mathrm{~b}$, with the exception that, in Experiments $2 \mathrm{a}$ and $2 \mathrm{~b}$, the photos were filtered for spatial frequency. Low-spatial frequency information was filtered-out from the photos in Experiment $2 \mathrm{a}$ and high-frequency information was filtered out in Experiment 2b (Fig. 1b and c, respectively). Spatial frequency information was filtered out using Photoshop CS (Adobe Systems, Palo Alto, CA). The high-frequency stimuli were created by applying the high-pass filter (radius 0.5 pixels). The low-frequency stimuli were created using the Gaussian blur filter (radius 5 pixels). This manipulation enabled us to filter out information about facial wrinkles in Experiment 2b, and conversely, to highlight the presence of wrinkles in Experiment 2a.

\section{Results and discussion}

The mean perceived age of neutral and smiling faces is presented in Fig. 3. As can be seen in the figure, the findings of Experiment 2 a replicate those found in Experiment 1. In particular, smiling faces were perceived as older compared with the same persons with a neutral expression $[\mathrm{t}(19)=4.59, P<$ .05 , partial eta square $=.53$ ). Conversely, in Experiment $2 \mathrm{~b}$, in which wrinkle-related information was filtered out, perceived age did not differ significantly between the neutral and the smiling faces $[\mathrm{t}(18)=0.41, P>.05$, partial eta square $=.01]$. To compare directly the effects on smile between the high and low frequency images, we entered the data into a mixed ANOVA design with expression (smile vs neutral) as a within-subject variable and experiment (2a vs $2 \mathrm{~b}$ ) as a between-subject variable. A main effect of expression [F(1, $37)=11.26, P<.01$, partial eta square $=.23$ ) indicated that smiling faces were perceived as older compared with neutral faces. More importantly, this effect was modulated by a significant expression $X$ experiment interaction $[\mathrm{F}(1,37)=7.53$, $P<.01$, partial eta square $=.17]$. This interaction indicates that the effect of smile on perceived age was significantly larger for the face images containing information about high spatial frequency compared low spatial frequency. The main effect of experiment was not significant $[\mathrm{F}(1,37)=2.87, P>.05$, partial eta square $=.07]$.

\section{Image-based analysis}

An image-based analysis was performed on the full-scale spectrum images of the database to complement the

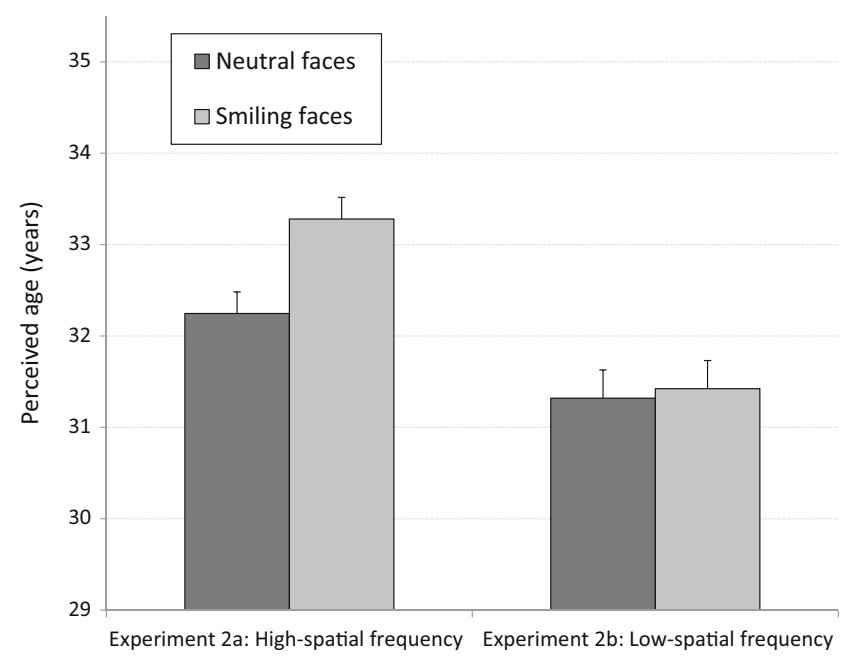

Fig. 3 Average perceived age for smiling and neutral faces in Experiments $2 \mathrm{a}-2 \mathrm{~b}$. Smiling faces were perceived as older compared to neutral faces when information on wrinkles was available for high spatial frequency images (Experiment 2a). When wrinkles were concealed (Experiment 2b, low spatial frequency images), smiling had no significant effects on age evaluations. Error bars represent $95 \%$ confidence intervals for within-subject designs (Jarmasz \& Hollands, 2009) 
behavioral analysis. The purpose of this analysis was to further test my prediction that wrinkles around the region of the eyes, manifested in the high spatial frequency information, are more pronounced in smiling compared to neutral faces. To this end, a fast Fourier transform (FFT) analysis was performed on the images using the MATLAB package. FFT allows the spatial aspects of the image to be decompressed to different frequency ranges. First, a rigid co-registration toolbox was used to co-register pairs of neutral and smiling photos of the same person. Analysis was focused on the general region of the eyes $(150 \times 50$ pixels $)$. We have then partitioned the selected regions to the rightmost and leftmost squares $(50 \times 50$ pixels $)$, which contained the regions of the right and left eyes for which wrinkles are predicted to be associated with smiles. A region in the middle square that contained the lower part of the nose served as a control region in that smiling was not predicted to elicit any increase in the amount of wrinkles in that region. Next, we performed the FFT analysis and computed the Fourier power spectrum slope for each image. This analysis was accomplished by computing the linear fit of the loglog relationship between the power spectrum and the spatial frequency. The value of the slope of each image reflects the relative amount of high compared to low spatial frequencies of the image. This analysis method has been used in the literature for facial images, with steeper slopes indicating enhanced low spatial frequencies and attenuated high spatial frequencies compared to shallower slopes (Blickhan, Kaufmann, Denzler, Schweinberger, \& Redies, 2011). It was therefore predicted that smiling faces would be accompanied with shallower slopes compared to neutral images of the same people. To complete the analysis, the slope values of the right and the left eyes were averaged for each neutral and smiling face. The results showed that the average slope of the neutral faces was indeed steeper $(\mathrm{S}=-3.68, \mathrm{SD}=0.62)$ compared to the slope of smiling faces of the same people $(\mathrm{S}=-3.34, \mathrm{SD}=$ 0.49 ). This difference was significant $[\mathrm{t}(79)=5.75, P<.001$, partial eta square $=.29$ ). As predicted, the corresponding differences between the slopes of the neutral and smiling faces for the control region of the bottom of the nose were not statistically different $(\mathrm{S}=-3.074, \mathrm{SD}=0.27 ; \mathrm{S}=-3.066$, $\mathrm{SD}=0.27$, respectively for neutral and smiling faces; $\mathrm{t}(79)=$ $0.35, P=.73$, partial eta square $=.002$ ) . These findings show that a larger power of high spatial frequencies is evident around the region of the eyes for smiling compared to neutral faces, probably due to the formation of wrinkles around this region.

The results of Experiments 2a and 2b show that the effect of smile on perceived age is based on fine-grained pictorial information, which contains information regarding the presence of wrinkles around the eyes. Such information is based on the high-spatial frequencies of the image. When the highspatial frequencies were graphically filtered out, smiling had no significant effect on perceived age. In Experiments $3 \mathrm{a}$ and
$3 b$, I further explored the nature of the effect of smile on perceived age, and provide an additional test for the idea that it is based on information from the region of the eyes.

\section{Experiments 3a-3b}

The purpose of Experiments $3 \mathrm{a}$ and $3 \mathrm{~b}$ was to provide an additional test for the idea that the aging effect of smile mainly resulted from the formation of wrinkles around the region of the eyes. To this purpose, I tested whether the effect would be modulated by the presentation of the top half of the face (that includes the eye region, Experiment 3a) compared to the bottom half of the face (that does not include the eye region, Experiment 3b).

\section{Method}

\section{Participants}

Twenty students from Ben Gurion University of the Negev (five male, mean age $=23$ years, $\mathrm{SD}=1.2$ years) participated in Experiment 2a and 20 different participants (three males) participated in Experiment 2b. Due to a registration error, the age of the participants in Experiment $3 \mathrm{~b}$ was not available. Nevertheless, these participants were recruited from the same student population as in the other experiments in this study.

\section{Design and procedure}

The design and procedure were similar to those used in Experiments 1 and 2. In Experiments $3 a$ and $3 b$, the fullspectrum facial stimuli that were presented in Experiment $1 \mathrm{c}$ were cropped graphically (horizontally at about the midline of the nose) for their top and bottom halves (Fig. 1d and e, respectively). This allowed the effects of wrinkles between the top half of the face, which contains wrinkles located in the region of the eyes, to be compared to the bottom half of the face, which contains information mainly about the region of the mouth and other features related to the formation of smiles.

\section{Results and discussion}

The mean perceived age of neutral and smiling faces in Experiments $3 \mathrm{a}$ and $3 \mathrm{~b}$ is presented in Fig. 4. As can be seen in the figure, the results for the top parts of the faces (Experiment 3a) replicate those found in Experiments 1 and 2, with smiling faces perceived as significantly older compared to neutral faces $[\mathrm{t}(19)=7.48, P<.01$, partial eta square $=.75]$. A similar effect, although of smaller magnitude, was found for the bottom part of the faces (Experiment 3b), $[\mathrm{t}(19)=3.06, P<.01$, partial eta square $=.33$ ]. This effect could have resulted from two possible sources. First, it is possible that some of the 


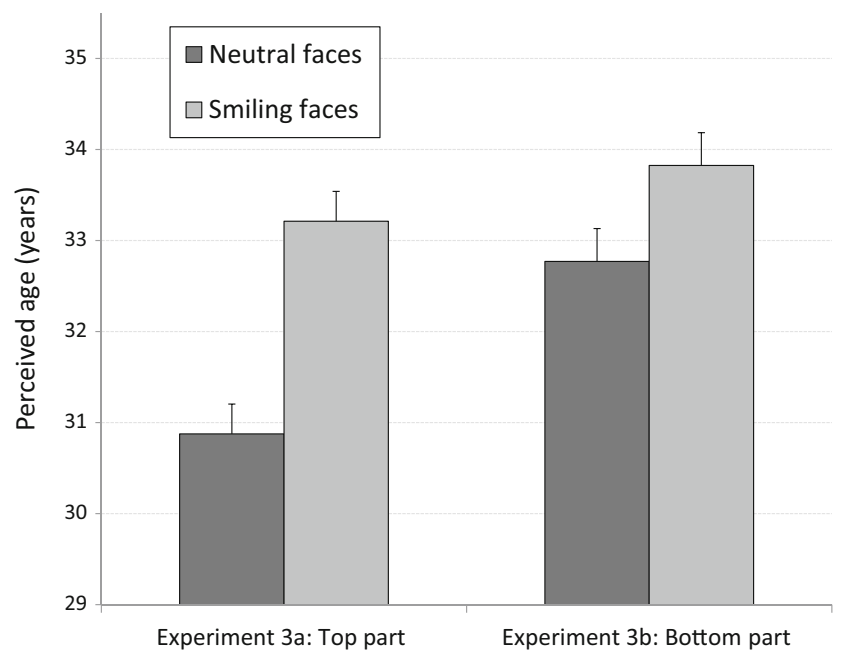

Fig. 4 Average perceived age for smiling and neutral faces in Experiments $3 \mathrm{a}-3 \mathrm{~b}$. As in previous experiments, smiling faces were perceived as older compared to neutral faces. The effect was significantly larger when information on wrinkles around the region of the eyes was available in the top parts of the faces (Experiment 3a) compared to when only the bottom parts of the faces were presented (Experiment 3b). Error bars represent $95 \%$ confidence intervals for within-subject designs (Jarmasz \& Hollands, 2009)

information about the wrinkles within the eye region also spreaded to the lower regions of the faces presented in Experiment $1 \mathrm{~b}$. In addition, it is also possible that smiling had an aging effect on the lower part of the faces due to the formation of some wrinkles around the region of the mouth (see Fig. 1e).

To test the idea if the aging effect of smiling was larger for the region of the eye compared to the region of the mouth, a direct comparison was made between the effects of smiling on the top and the bottom parts of face, using a mixed ANOVA design with expression (smile vs neutral) as a within-subject variable and experiment ( $3 a$ vs $3 b$ ) as a between-subject variable. A main effect of expression $[\mathrm{F}(1,38)=57.5, P<.01$, partial eta square $=.58$ ) indicated that smiling faces were perceived as older compared with neutral faces. More important, this effect was modulated by a significant expression $\mathrm{X}$ experiment interaction $[\mathrm{F}(1,38)=7.61, P<.01$, partial eta square $=.17$ ). This interaction indicates that the effect of smile on perceived age was significantly larger for the top part of the face, which contains the region of the eyes, compared with the bottom part of the face. The main effect of the experiment was not significant $[\mathrm{F}(1,38)=3.47, P>.05$, partial eta square $=$ $.084)$. Together with the findings of Experiments $2 a$ and $2 b$, these findings strongly suggest that the aging effect of smile originates from the creation of wrinkles around the region of the eyes during smiles. When such fine-grained information was filtered out in Experiment $2 b$, we found no effects of smile on performance. In a similar vein, when age judgments were based only on the bottom part of the face, which does not contain information on wrinkles, the effects of smile on perceived age was significantly reduced.

\section{General discussion}

The findings of Experiments 1-3 are straightforward: When people smile, they are perceived as older than when they express a neutral emotion. This provides the first empirical demonstration that, contrary to what has been commonly assumed, and contrary to what has been suggested in a previous study (Voelkle et al. 2012), smiling makes people look older rather than younger.

As discussed in the Introduction, only one previous study has looked at the effect of smiling (as well as of other facial expressions) on perceived age (Voelkle et al. 2012). Interestingly, the authors of this study latter concluded that smiling expression leads to faces being perceived as younger rather than older. However, a closer look at the results reveals that the findings did not show any statistical differences between faces presented in smiling and neutral expressions. Faces bearing these two expressions were evaluated to be approximately of the same age. Yet, faces bearing other expressions, such as fear, disgust, and anger, were perceived as older compared to faces bearing neutral and smiling expressions. As discussed above, Voelkle et al.'s (2012) design, in which photos of the same person were presented repeatedly in the experiment for age evaluation could have biased overt age evaluation in their study. The question of how and whether different expressions, beyond smiling, affect perceived facial age deserves further examination in future research.

Although the present findings provide strong support for the idea that the aging effect of smiling originates from the formation of wrinkles around the region of the eyes, alternative explanations are also possible. For example, smiling may lead to narrowing of the eyes, which has been suggested to result in facial aging (Marsh, Adams, \& Kleck, 2005). Although the present findings cannot completely refute such an alternative account, the results of Experiment 2b, in which low-frequency images were presented, do not support the idea that the aging effect I report here can be attributed to narrowing of the eyes. In particular, filtering out the high spatial frequencies from the images in Experiment $2 b$ leads to the removal of wrinkles, but did not abolish the possible effects of eye narrowing following smiles. Yet, when wrinkles were removed from the images in Experiment 2b, smiling did not lead to faces being perceived as older, although the eyes were still narrowed for smiling compared to neutral faces. Therefore, the results of Experiment 2, together with those found in Experiment 3 provide support for the idea that smiling faces were perceived as older than neutral faces, primarily due to the formation of wrinkles around the region of the eyes.

The robust effect of aging due was found in the present study across different face databases and across different experiments. Note, however, that the faces presented in all databases used throughout this study were of young adults, in the 
age range of 20-40 years. The question of whether smiling would show similar effects of aging for faces belonging to older (as well as younger) age groups is relevant but is beyond the scope of the present study. We plan to study the generality of the results across other age groups of faces as well as across different participant age groups in future studies in my laboratory.

Although facial age is considered to be one of the most immediate aspects of information people extract when viewing a new, unfamiliar person (George \& Hole, 1998), the task of extracting the actual age of a person based on visual information alone is not straight forward. In particular, previous research has shown that the actual age of a person is not necessarily reflected on his/her face; although there are several physical markers of facial age, such as facial shape, texture, color, and wrinkles (Burt \& Perrett, 1995; Lai, Oruc, \& Barton, 2013; Porcheron, Mauger, \& Russell, 2013), different people age differently. Aging is determined largely by genetic aspects, personal health history, and exposure to sun (Gunn et al. 2009; Mark et al. 1980). Therefore, it is well accepted that, although information on age is readily extracted, people are not accurate in determining the actual age of the person based on his/her appearance alone (Voelkle et al. 2012). Such inaccuracy inevitably leads to potential effects of irrelevant information on age evaluations (Karnadewi \& Lipp, 2011). The present results show that temporary changes in facial expression, such as smile, lead to consistent, directional biases in judging a person's age.

Acknowledgments I thank Daniel Algom for his helpful comments on an earlier version of the manuscript, Svetlana Lubinsky for her help with the image analysis, and Gal Namdar and Reli Lifshitz for their help with running the experiments.

\section{References}

Blickhan, M., Kaufmann, J. M., Denzler, J., Schweinberger, S. R., \& Redies, C. (2011). 1/f(p) Characteristics of the Fourier power spectrum affects ERP correlates of face learning and recognition. Biological Psychology, 88(2-3), 204-214.

Burt, D. M., \& Perrett, D. I. (1995). Perception of age in adult Caucasian male faces: Computer graphic manipulation of shape and colour information. Proceedings of the Royal Society B: Biological Sciences, 259(1355), 137-143.

Dagovitch, Y., \& Ganel, T. (2010). Effects of facial identity on age judgments: Evidence from repetition priming. Experimental Psychology, 57(5), 390-397.

Ebner, N. C. (2008). Age of face matters: Age-group differences in ratings of young and old faces. Behavior Research Methods, 40(1), 130-136.

Ebner, N. C., Riediger, M., \& Lindenberger, U. (2010). FACES-a database of facial expressions in young, middle-aged, and older women and men: Development and validation. Behavior Research Methods, 42(1), 351-362.

Ganel, T., \& Goshen-Gottstein, Y. (2002). Perceptual integrality of sex and identity of faces: Further evidence for the single-route hypothesis. Journal of Experimental Psychology: Human Perception and Performance, 28(4), 854-867.

Ganel, T., \& Goshen-Gottstein, Y. (2004). Effects of familiarity on the perceptual integrality of the identity and expression of faces: The parallel-route hypothesis revisited. Journal of Experimental Psychology: Human Perception and Performance, 30(3), 583-597.

Ganel, T., Goshen-Gottstein, Y., \& Goodale, M. A. (2005). Interactions between the processing of gaze direction and facial expression. Vision Research, 45(9), 1191-1200.

George, P. A., \& Hole, G. J. (1998). Recognising the ageing face: The role of age in face processing. Perception, 27(9), 1123-1124.

Gunn, D. A., Rexbye, H., Griffiths, C. E. M., Murray, P. G., Fereday, A., Cat, S. D., ... Christensen, K. (2009). Why some women look young for their age. PLoS One, 4(12), e8021.

Jarmasz, J., \& Hollands, J. G. (2009). Confidence intervals in repeatedmeasures designs: The number of observations principle. Canadian Journal of Experimental Psychology/Revue canadienne de psychologie expérimentale, 63(2), 124-138

Jones, B. C., Debruine, L. M., Little, A. C., Conway, C. A., \& Feinberg, D. R. (2006). Integrating gaze direction and expression in preferences for attractive faces. Psychological Science, 17(7), 588-591.

Karnadewi, F., \& Lipp, O. V. (2011). The processing of invariant and variant face cues in the Garner Paradigm. Emotion, 11(3), 563-571.

Lai, M., Oruc, I., \& Barton, J. J. (2013). The role of skin texture and facial shape in representations of age and identity. Cortex, 49(1), 252-265.

Lundqvist, D., Flykt, A., \& Öhman, A. (1998). The Karolinska Directed Emotional Faces - KDEF, CD ROM from Department of Clinical Neuroscience, Psychology section, Karolinska Institutet.

Mark, L. S., Pittenger, J. B., Hines, H., Carello, C., Shaw, R. E., \& Todd, J. T. (1980). Wrinkling and head shape as coordinated sources of age-level information. Perception \& Psychophysics, 27(2), 117124.

Marsh, A. A., Adams, R. B., Jr., \& Kleck, R. E. (2005). Why do fear and anger look the way they do? Form and social function in facial expressions. Personality and Social Psychology Bulletin, 31, 73-86.

Minear, M., \& Park, D. C. (2004). A lifespan database of adult facial stimuli. Behavior Research Methods, Instruments, \& Computers, 36(4), 630-633.

Otta, E., Lira, B. B. P., Delevati, N. M., Cesar, O. P., \& Pires, C. S. G. (1994). The effect of smiling and of head tilting on person perception. Journal of Psychology, 128(3), 323-331.

Porcheron, A., Mauger, E., \& Russell, R. (2013). Aspects of facial contrast decrease with age and are cues for age perception. PLoS One, $8(3)$, e57985.

Root, A. A., \& Stephens, J. A. (2003). Organization of the central control of muscles of facial expression in man. Journal of Physiology, 549, 289-298.

Schweinberger, S. R., \& Soukup, G. R. (1998). Asymmetric relationships among perceptions of facial identity, emotion, and facial speech. Journal of Experimental Psychology Human Perception \& Performance, 24(6), 1748-1765.

Tsukiura, T., \& Cabeza, R. (2008). Orbitofrontal and hippocampal contributions to memory for face-name associations: The rewarding power of a smile. Neuropsychologia, 46(9), 2310-2319.

Voelkle, M. C., Ebner, N. C., Lindenberger, U., \& Riediger, M. (2012). Let me guess how old you are: Effects of age, gender, and facial expression on perceptions of age. Psychology and Aging, 27(2), 265-277. 